\title{
A new algorithm for available transfer capability computation
}

\author{
C.-Y. Li, C.-W. Liu* \\ Department of Electrical Engineering, National Taiwan University, Taipei, Taiwan
}

\begin{abstract}
This paper proposes a new algorithm that calculates the Available Transfer Capability (ATC) accurately. The accuracy of computing ATC is important to ensure the security of system operation. On the hand, we devise the maximum area concept for the allocation of simultaneous ATC. This concept decides maximum area capability inside the security region by considering the shape of security boundary. The proposed concept is novel because the other allocation methods only use the maximum transfer amount to compute ATC. The proposed concept improves the efficiency of transmission facility use and makes the power interchange more flexible. (C) 2002 Elsevier Science Ltd. All rights reserved.
\end{abstract}

Keywords: ATC; Open access; Deregulation

\section{Introduction}

In 1996, the Federal Energy Regulatory Commission (FERC) mandated the regulatory orders, including order numbers 888 and 889 in the deregulation framework. The order claims the public utility to open their transmission grid for use by market participants. For the purpose of nondiscriminatory transmission open access, a sufficient message should be posted to the grid user on the Open Access Same-time Information System (OASIS). The Available Transfer Capability (ATC) is one term of such an ancillary service. The ATC is an amount of the transfer capability remaining in the physical transmission line for further commercial transaction. The NERC also develops the ATC principle in accordance with the provision of the FERC rule [1]. ATC is defined as the Total Transfer Capability (TTC) less than the Transmission Reliability Margin (TRM), and less than the sum of existing commitments and Capacity Benefit Margin (CBM) in the principle.

Because the power system is a large-scale nonlinear network and generation-load flow is system wide relevant, the transaction could happen at any bus node in the deregulation system. All of these make it difficult to measure the accurate transfer capability. Although, there have been several papers [2-12] dealing with this topic, most of them are under assumption conditions to compute the approximate transfer capability. Therefore, they must certainly reserve more TRM due to the inaccurate transfer

\footnotetext{
* Corresponding author. Tel.: +886-2-2363-5251; fax: +886-2-2371909.

E-mail address: cwliu@cc.ee.ntu.edu.tw (C.-W. Liu).
}

capability solution. In this paper, we develop the sensitivitybased algorithm that pins down transfer capability accurately. The sensitivity index has been successfully applied in power systems for the control of system voltage and economic dispatch control [11-14]. Here, we use the sensitivity to search for ATC and identify critical lines at the same time. For the purpose of sufficient use of transmission capacity and fair transaction, the maximum area concept is devised.

There are five sections in this paper. In Section 2, the maximum area concept is devised and the sensitivitybased algorithm is presented. In Section 3, we apply the proposed algorithm to a 6-bus system mentioned in Ref. [3] and compare its results. The IEEE 30-bus is also investigated there. In Section 4, the strategy of allocation of transfer capability is discussed.

\section{The proposed sensitivity-based algorithm}

There have been several problem formulations for ATC. In this section, we present a simple and static ATC formulation for illustrating the proposed sensitivity-based algorithm. However, we would like to emphasize that the sensitivity-based algorithm can be easily extended to more general problem formulations, such as including contingency constraints, transient stability constraints and voltage stability constraints, etc.

\subsection{ATC problem formulation}

Denote the bus set of entire system by $\Phi$, the transaction 


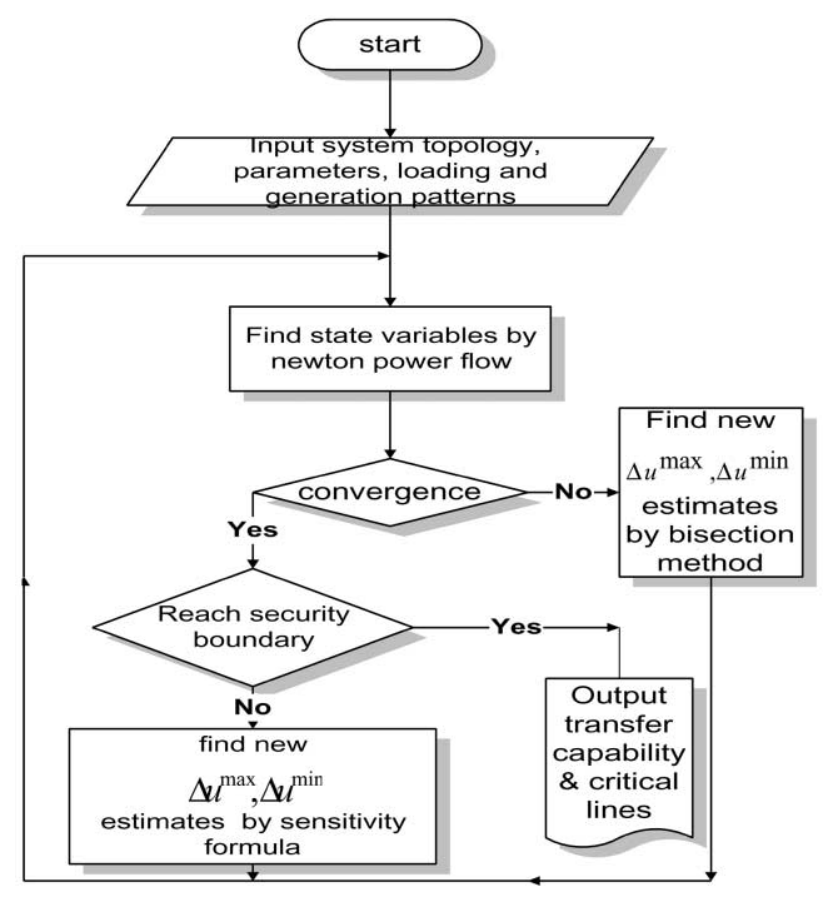

Fig. 1. Flow chart of calculation of 1-dimension ATC by sensitivity-based algorithm.

bus set by $\Omega$. Then ATC is formulated as the extreme values of the incremental power capacity of the transaction buses under the condition that the security is assured. In mathematical form, ATC is expressed as:

Find $\quad \max \left(\Delta u_{k}\right)$ and $\min \left(\Delta u_{k}\right) \quad \forall k \in \Omega$

subject to

$u_{k}+\min \left(\Delta u_{k}\right) \leq u_{k} \leq u_{k}+\max \left(\Delta u_{k}\right) \quad \forall k \in \Omega$

$f(x, u, w)=0$

$\delta \leq \theta_{i j} \leq \bar{\delta} \quad \forall i, j \in \Phi$

$\underline{V} \leq V \leq \bar{V}$

$\underline{P} \leq P \leq \bar{P}$

$Q \leq Q \leq \bar{Q}$

where $x$ is state vector including bus voltage phasors, $u$ is vector including bus power injection and demand, $w$ is parameter vector including network parameters. The upper and lower bar mean maximum and minimum value, respectively. The $f(x, u, w)$ is load flow equation. Eqs. (4)-(7) are inequality constraints. $\theta_{i j}$ is phase difference. $\delta$ is phase limit due to thermal line constraint.

\subsection{Computation of ATC for single bus transaction (1- dimension ATC)}

For the sake of ease of deriving our algorithm, we merely deal with real power transfer capability i.e. $\Delta u_{k}=\Delta p_{k}$ and only consider thermal line constraint.

The first order sensitivity for load flow equation, Eq. (3), is

$\Delta x=S_{u} \cdot \Delta u+S_{w} \cdot \Delta w$

where $S_{u}=-f_{x}^{-1} \cdot f_{u}, S_{w}=-f_{x}^{-1} f_{u} f_{x}, f_{u}, f_{w}$ are partial derivatives of $f$ with respect to variable $x, u, w$.

Assume we consider the static power system and evaluate ATC of single bus transaction, then we have the following simplified equation.

$\Delta w=0 \quad f_{u}=-I(I:$ Identity matrix $)$

$\Delta x=S_{u} \cdot \Delta u=f_{x}^{-1} \cdot \Delta u$

We notice that $f x$ is Jacobian matrix. Hence, the sensitivity index, $f_{x}^{-1}$, is available for computation use if Newton method is applied to solve power flow.

In order to get phase difference column vector, we multiply the incidence matrix $A^{T}$ [14] on both sides of Eq. (9).

$A^{T} \cdot \Delta x=A^{T} \cdot f_{x}^{-1} \cdot \Delta u$

Set $A^{T}(j, i)=1, A^{T}(j, k)=-1$, the other elements of $A^{T}$ are zero. Let $\Delta x=(\Delta \theta, \Delta v)^{T}$ denote the incremental phase and voltage vector for all the buses. Substitutes all elements of column vector $\Delta u$ by zero except the $j$-th element by $\Delta p_{j, 1}$. Thus, we obtain the following Eq. (10b).

$\Delta \theta_{i k}=\left[\left(S_{u}\right)_{i j}-\left(S_{u}\right)_{i k}\right] \times \Delta p_{j, 1}=\left[\left(f_{x}^{-1}\right)_{i j}-\left(f_{x}^{-1}\right)_{k j}\right] \times \Delta p_{j, 1}$

The transfer capability is the maximum and minimum incremental power subject to load flow equation and security constraints. Apply Eq. (10b), we obtain the following $\Delta p_{j, 1}^{\max }$ and $\Delta p_{j, 1}^{\min }$ estimate equations:

$\Delta P_{j, 1}^{\max } \approx \min _{(i, k)}\left[\frac{\bar{\delta}-\theta_{i j}}{\left(f_{x}^{-1}\right)_{i j}-\left(f_{x}^{-1}\right)_{k j}}\right]$

$\Delta P_{j, 1}^{\min } \approx \min _{(i, k)}\left[\frac{\theta_{i j}-\underline{\delta}}{\left(f_{x}^{-1}\right)_{i j}-\left(f_{x}^{-1}\right)_{k j}}\right]$

$\forall(i, k) \in$ transmission line branches

Since Eqs. (11) and (12) provide us the estimates of $\Delta p_{j, i}^{\max }$ and $\Delta p_{j, i}^{\min }$, we apply the Newton's iteration to solve power flow equation using these estimates as initial conditions, and examine whether thermal limit of any branch is violated. That is, use Eqs. (11) and (12) to predict the $\Delta P j$ value of next step. The iteration continues until the thermal constraint is violated. On the other side, the predictive 




Fig. 2. The geometry interpretation of maximum area concept for 2-dimension ATC

value of Eqs. (11) and (12) maybe lie outside the convergent region of power flow iteration. Then, we use bisection method to correct estimates, $\Delta p_{j, i}^{\max }$ and $\Delta p_{j, i}^{\min }$. Thus

$\left(\Delta P_{j}\right)^{r+1}=\left[\left(\Delta p_{j}\right)^{r}+\left(\Delta p_{j}\right)^{r-1}\right] / 2$

Assume the divergence happens at the $r$-th iteration, the $(r+1)$-th $\Delta p_{j}$ is evaluated by bisection method. The critical lines that reach the extreme thermal constraint boundary are just the branches where the $\Delta p_{j, i}^{\max }$ and $\Delta p_{j, i}^{\min }$ occur. Thus, the critical lines of $\mathrm{j}$-bus ATC are identified.

The algorithm is shown in Fig. 1.

\subsection{Computation of ATC for multiple buses transaction (multi-dimension ATC)}

Again, we only consider real power transfer capability and thermal line constraint. Substituting the transaction bus $\Delta p$ in vector $\Delta u$, the algorithm can be expanded to compute ATC for multiple buses. Here we introduce the maximum area concept that bases on the fair transaction and the sufficient use of transmission line. We use two buses transaction to explain the method briefly.

Assume both the $m$-th bus and the $n$-th bus requires transfer capability evaluation. Then substitute all elements of column vector $\Delta u$ of Eq. (10a) by zero, except the $m$-th element and the $n$-th element by $\Delta p_{m, 2}$ and $\Delta p_{n, 2}$, respectively. Then the phase difference for branch $(i, k)$ is given by

$\Delta \theta_{i k}=\left[\left(S_{u}\right)_{i m}-\left(S_{u}\right)_{k m}\right] \Delta P_{m, 2}+\left[\left(S_{u}\right)_{i n}-\left(S_{u}\right)_{k n}\right] \Delta P_{n, 2}$

Assume the critical line of maximum transfer capability happens at branch $(i, k)$.

Set

$\left(\Delta \theta_{i k}\right)^{\max }=\delta^{\max }-\theta_{i k}$

Denote 2-dimension ATC by $\Delta p_{m, 2}^{\max }, \Delta p_{m, 2}^{\min }$ and $\Delta p_{n, 2}^{\max }$, and $\Delta p_{n, 2}^{\min }$, respectively. They should satisfy Eq. (14). That is

$$
\begin{aligned}
& \left(\Delta \theta_{i k}\right)^{\max }\left[\left(S_{u}\right)_{i m}-\left(S_{u}\right)_{k m}\right] \Delta P_{m, 2}^{\max }+\left[\left(S_{u}\right)_{i n}-\left(S_{u}\right)_{k n}\right] \Delta P_{n, 2}^{\max } \\
& \left(\Delta \theta_{i k}\right)^{\min }\left[\left(S_{u}\right)_{i m}-\left(S_{u}\right)_{k m}\right] \Delta P_{m, 2}^{\min }+\left[\left(S_{u}\right)_{i n}-\left(S_{u}\right)_{k n}\right] \Delta P_{n, 2}^{\min }
\end{aligned}
$$

Set

$\Delta P_{m}^{0}=\left(\Delta \theta_{i k}\right)^{\max } /\left[\left(S_{u}\right)_{i m}-\left(S_{u}\right)_{k m}\right]$

$\Delta P_{m}^{0}=\left(\Delta \theta_{i k}\right)^{\max } /\left[\left(S_{u}\right)_{i n}-\left(S_{u}\right)_{k n}\right]$

Compare Eqs. (16) and (17) with Eq. (11), we find they are similar. We would like to introduce the maximum area concept for multi-dimension ATC by geometry. Let us first denote 1-dimension ATC of bus $m$ and bus $n$ by $\Delta p_{m, 1}^{\max }$ and $\Delta p_{n, 1}^{\max }$. Then approximate $\Delta p_{m}^{0}$ and $\Delta p_{n}^{0}$ by $\Delta p_{m, 1}^{\max }$ and $\Delta p_{n, 1}^{\max }$, respectively. Then Eq. (15a) is rearranged as follows:

$1-\frac{\Delta P_{m, 2}^{\max }}{\Delta P_{m, 1}^{\max }}-\frac{\Delta P_{n, 2}^{\max }}{\Delta P_{n, 1}^{\max }}=0$

Set the objective function as follows:

$\operatorname{Area}\left(\Delta p_{m, 2}^{\max }, \Delta p_{n, 2}^{\max }\right)=\Delta p_{m, 2}^{\max } \times \Delta p_{n, 2}^{\max }$

Find the extreme value of Eq. (19) under the constraint Eq. (18) by LaGrange multiplier method. We obtain the following equation.

$\frac{\Delta P_{n, 2}^{\max }}{\Delta P_{m, 2}^{\max }}=\frac{\Delta P_{n, 1}^{\max }}{\Delta P_{m, 1}^{\max }}=K_{1}$

Substituting the maximum area relation Eq. (20) into (15), we will reduce two dimension problem to one dimension problem. Thus

$\Delta p_{m, 2}^{\max }={ }_{(i, k)}^{\min } \frac{\left(\Delta \theta_{i k}\right)^{\max }}{\left[\left(S_{u}\right)_{i m}-\left(S_{u}\right)_{k m}\right]+K_{1} \cdot\left[\left(S_{u}\right)_{i n}-\left(S_{u}\right)_{k n}\right]}$

$\Delta p_{n, 2}^{\max }=K_{1} \times \Delta p_{m, 2}^{\max }$

$\forall(i, k) \in$ transmission line branches

By the same way, we have the similar equations for the $\Delta p_{m, 2}^{\min }$ and $\Delta p_{n, 2}^{\min }$, respectively

$\frac{\Delta P_{n, 2}^{\min }}{\Delta P_{m, 2}^{\min }}=\frac{\Delta P_{n, 1}^{\min }}{\Delta P_{m, 1}^{\min }}=K_{2}$

$\Delta p_{m, 2}^{\min }=\min _{(\mathrm{i}, \mathrm{k})} \frac{\left(\Delta \theta_{i k}\right)^{\min }}{\left[\left(S_{u}\right)_{i m}-\left(S_{u}\right)_{k m}\right]+K_{2} \cdot\left[\left(S_{u}\right)_{i n}-\left(S_{u}\right)_{k n}\right]}$

$\Delta p_{n, 2}^{\min }=K_{2} \times \Delta p_{m, 2}^{\min }$

Because both Eqs. (21) and (23) are only 1-dimension ATC problem, we can use the same algorithm of Fig. 1 to compute the extreme capacity, $\Delta p_{m, 2}^{\max }, \Delta p_{m, 2}^{\min }$, for bus $m$ at first. Subsequently, substitute the extreme capacity of bus $m$ into Eqs. (20) and (22) to find extreme capacity $\Delta p_{n, 2}^{\max }, \Delta p_{n, 2}^{\min }$ of bus $n$. The above procedure is shown in Fig. 2. The 2-dimension ATC is obtained through the constant slope path of Eqs. (20) and (22). Thus the maximum area ATC is obtained by following the constant slope 
Table 1

The calculated 1-dimension ATC for 6-bus example bus with phase difference constraint, \pm 0.3491

\begin{tabular}{ll}
\hline Line (1-0), $\Delta P_{1}^{\min }=-0.3069$ & Line (1-0), $\Delta P_{1}^{\max }=1.8528$ \\
Line (3-0), $\Delta P_{2}^{\min }=-0.4845$ & Line (3-0), $\Delta P_{2}^{\max }=1.9617$ \\
Line (3-0), $\Delta P_{3}^{\min }=-0.5192$ & Line (3-0), $\Delta P_{3}^{\max }=2.0024$ \\
Line (1-0), $\Delta P_{4}^{\min }=-0.3218$ & Line (1-0), $\Delta P_{4}^{\max }=0.7820$ \\
Line (1-0), $\Delta P_{5}^{\min }=-0.4312$ & Line (1-0), $\Delta P_{5}^{\max }=0.3636$
\end{tabular}

of line $\overleftrightarrow{A O}, \overleftrightarrow{C O}$, until points $\mathrm{B}$ and $\mathrm{D}$ reach the security boundary, respectively.

Denote the transaction buses by set $\Omega$ for multi-dimension ATC, then the general form of ATC of multiple buses is the following procedure.

If the $m$-th bus belongs to set $\Omega$, and $R_{i k}$ is the subtraction row vector of $S_{u}$ where

$R_{i k}=\left(S_{u}\right)_{i}-\left(S_{u}\right)_{k}$

and $P^{\max }, P^{\min }$ are the column vectors with the elements of zero, except that

$\left(P^{\max }\right)_{i}=\Delta P_{i, 1}^{\max } / \Delta P_{m, 1}^{\max }$

$\left(P^{\min }\right)_{i}=\Delta P_{i, 1}^{\min } / \Delta P_{m, 1}^{\min }$

$\forall \mathrm{i} \in \Omega$

where $p_{i, 2}^{\max }$ and $p_{i, 1}^{\min }$ mean the 1-dimension ATC of the $i$-th bus. Then ATC is given as:

for the $m$-th bus:

$\Delta p_{m}^{\max }={ }_{(i, k)}^{\min }\left(\Delta \theta_{i k}\right)^{\max } / R_{i k} \cdot P^{\max }$

$\Delta p_{m}^{\min }={ }_{(i, k)}^{\min }\left(\Delta \theta_{i k}\right)^{\min } / R_{i k} \cdot P^{\min }$

$\forall(\mathrm{i}, \mathrm{k})$ denote the grid branches $m \in \Omega$ for other buses

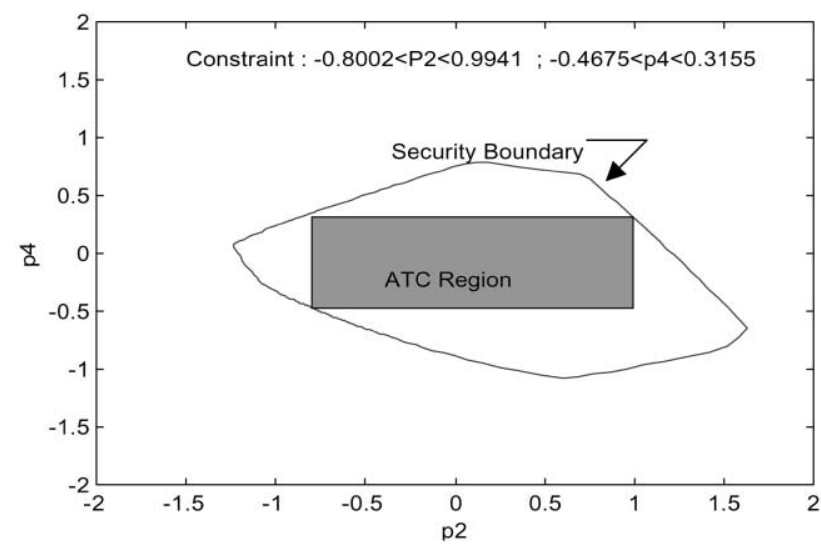

Fig. 3. The calculated 2-dimension ATC for bus 2 and bus 4 in 6-bus system by maximum area algorithm.

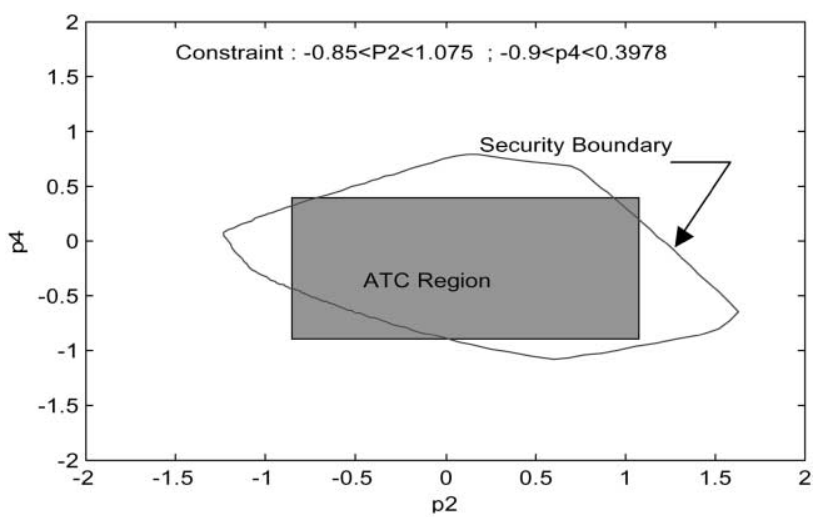

Fig. 4. The calculated 2-dimension ATC for bus 2 and bus 4 in 6-bus system by monotonicity method.

except the $m$-th bus:

$\Delta P_{i}^{\max }=\left(P^{\max }\right)_{i} \times \Delta P_{m}^{\max }$

$\Delta P_{i}^{\min }=\left(P^{\min }\right)_{i} \times \Delta P_{m}^{\min }$

$\forall i \in \Omega, i \neq m$

\section{Simulation results}

In this section, we tested our algorithm with 6-bus system and IEEE 30-bus system. The result of 6-bus system is compared with the result of Ref. [3] in which the same example is computed by the monotonicity method. These results encourage us as to the feasibility of the proposed algorithm for power system.

\subsection{The ATC of single bus (1-dimension ATC) for 6-bus system}

The network of 6-bus system, the current operating point and network parameters are shown in Appendix A. Assume we are interested in available transfer capability of single bus. Substitute proper incremental power in Eqs. (11) and (12), respectively, and apply the sensitivity-based algorithm as shown in Fig. 1, then we get the 1-dimension ATC of each bus. The results are listed in Table 1 . The critical line is shown in the parentheses. Notice that the ATC of one dimension is large.

\subsection{The ATC of multiple buses transaction (multi-dimension ATC) for 6-bus system}

The sensitivity-based algorithm of multiple buses is to reduce multiple dimension computation into one dimension computation under maximum area concept. As long as the extreme capability of one bus has been obtained by Eqs. (21) and (23a), all of the relevant extreme values of the others can be evaluated by Eqs. (21b) and (23b) simultaneously. For depicting briefly, we only shown 2-dimension ATC of bus 2 and bus 4 in Fig. 3. Here, we will compare our results 


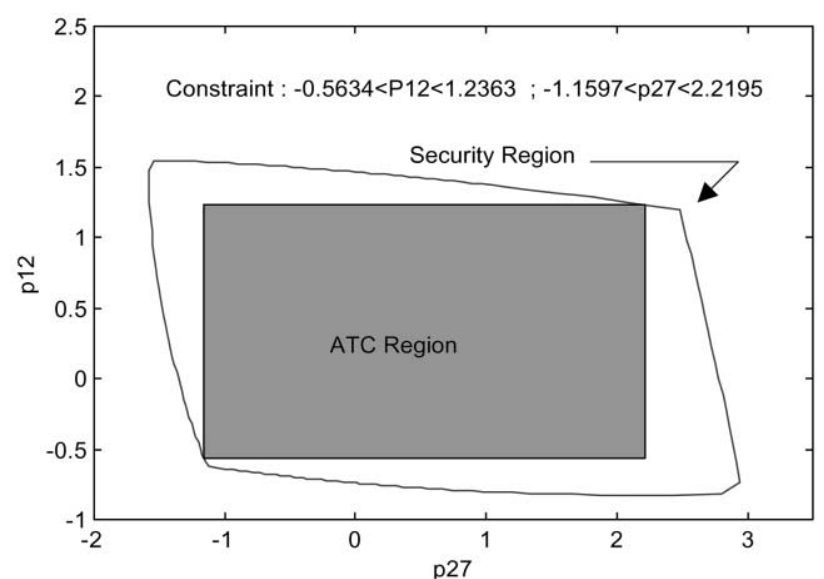

Fig. 5. The calculated 2-dimension ATC for bus 2 and bus 4 in 6-bus system by maximum area algorithm.

with the other, called monotonicity method [15-17] using the same 6-bus system. For the comparison, we begin with the same operating point, network parameters and thermal constraint. Then the 2-dimension ATC of bus 2 and bus 4 computed by monotonicity method is shown in Fig. 4. Obviously, the calculated ATC is not secure in which some parts of ATC lie outside the security boundary. The 2-dimension ATC for other buses by monotonicity method also has the same drawbacks.

\subsection{Compute ATC for IEEE 30-bus [18]}

We verify the sensitivity-based algorithm by IEEE 30bus system (Appendix B) to prove our algorithm is potential for larger power systems.

Assume we will compute 2-dimension ATC between two distant buses, bus 12 and bus 27. At first, begin with 1dimension ATC computation by Eqs. (11) and (12). Then the critical lines of the maximum and minimum capacity of each single bus are line 4-12 and line 27-28 for bus 12 and bus 27, respectively. The 1-dimension ATC is shown as follows.

$\Delta P_{12}^{\min }=-1.3569, \quad \Delta P_{12}^{\min }=-2.7818$
$\Delta P_{27}^{\min }=-0.7297, \quad \Delta P_{27}^{\min }=-1.4751$

Thermal line constraint $=0.3032$

The 2-dimension ATC based on maximum area method is shown in Fig. 5. Note that the computed ATC is secure and maximum in some sense.

\section{Discussion}

There are several patterns for allocation of multidimension ATC. We outline it by two generator buses in Fig. 6. As the drawing shows, there are infinite points between point A,B security boundary. It is essential to discuss which pattern is fair and plausible.

Because the entire security boundary is unknown, we can only inform the market participants' ATC message through the data link network. That is a rectangle region inside the security boundary. The rectangle region depends on the current operation condition. It is more flexible for dispatch demand if the region is as broad as possible. The maximum area concept supports the requirement, as shown at point $\mathrm{C}$. The economic consideration supports another choice to allocate, as shown at point D. But as there are many factors affecting the bidding price in the deregulation system, the price is hard to forecast. It may be smaller region than the maximum area one that is available for allocation. We notice that the proposed concept has considered the maximum area transaction inside the security shape. The security shape is composed of the interesting transaction buses $\Omega$. The concept differs from the others in that only the maximum transfer capability on the received bus, as bilateral transfer capacity (BITC) mentioned in paper [19].

Another benefit for the maximum area concept is fair allocation, due to the multi-dimension ATC is allocated by its 1-dimension ATC, respectively. The 1-dimension ATC denotes the owner right of the available transmission at that bus. So it is fair to allocate the multi-dimension ATC by 1-dimension ATC. Anyway, the maximum area supports

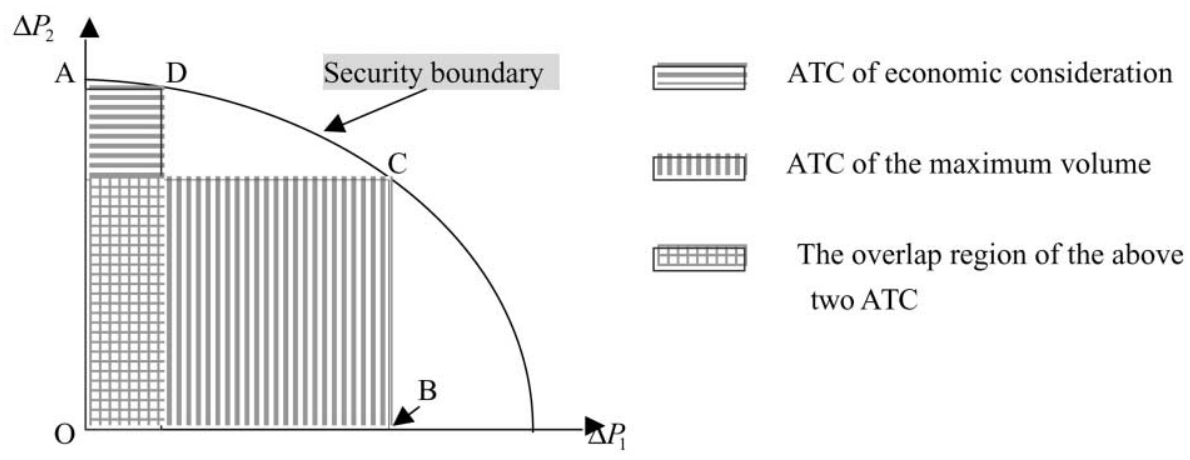

Fig. 6. The allocation problem for two generator buses. 
a new method for capacity allocation for the purpose of sufficient use of transmission grid. The essence of maximum area concept will be more important when the transaction service is weak and no more facility of grid can be expanded in the short term.

\section{Conclusions}

We proposed the maximum area concept and the sensitivity-based algorithm to meet the secure ATC. Beside that, we can also clearly identify which lines are critical. Simulation tests on 6-bus system and IEEE 30-bus system give us encouraging results.

Finally, we inspect the allocation of transfer capability. Because the more flexible ATC is desired, the maximum area concept meets the requirement. The other benefit is fair consideration. The evaluated ATC is secure enough to assure reliable operation within the ATC region and the region of ATC is the maximum in some sense.

\section{Appendix A. The 6-bus system}

Current operating conditions (in per unit) are as shown below:

\begin{tabular}{llrrr}
\hline Bus type & $\left|E_{i}\right|$ & \multicolumn{1}{l}{$\angle E_{i}$} & $\mathrm{P}$ & \multicolumn{1}{l}{$\mathrm{Q}$} \\
\hline 0 slack bus & 1.0500 & 0.0000 & 0.98169 & 0.46156 \\
1 PQ bus & 0.9188 & -0.2260 & -0.52500 & -0.05000 \\
2 PQ bus & 0.9213 & -0.2360 & -0.55000 & -0.13000 \\
3 PQ bus & 0.9367 & -0.1763 & 0.00000 & 0.00000 \\
4 PQ bus & 0.9045 & -0.2330 & -0.30000 & -0.18000 \\
5 PV bus & 1.1000 & -0.0910 & 0.50079 & 0.19800 \\
\hline
\end{tabular}

Network parameters (in per unit) are shown in the following table:

\begin{tabular}{lll}
\hline Line destination & Resistance & Reactance \\
\hline $0-1$ & 0.123 & 0.518 \\
$0-3$ & 0.08 & 0.370 \\
$1-3$ & 0.097 & 0.407 \\
$1-4$ & 0.000 & 0.300 \\
$2-3$ & 0.000 & 0.133 \\
$2-5$ & 0.723 & 1.050 \\
$4-5$ & 0.282 & 0.640 \\
\hline
\end{tabular}

\section{Appendix B. The IEEE 30-bus system}

Impedance and line-charging data (impedance and line-

\section{6-bus system}

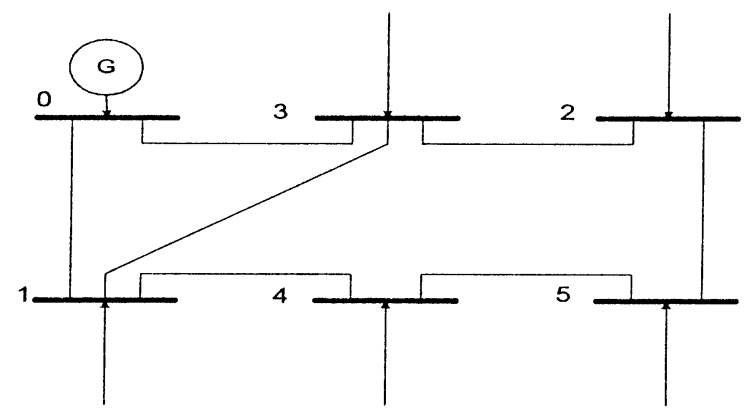

charging data in p.u. on $100,000 \mathrm{kVA}$ base) are shown in the following table:

\begin{tabular}{llll}
\hline $\begin{array}{l}\text { Line } \\
\text { designation }\end{array}$ & Resistance & Reactance & Line-charging \\
& p.u. & p.u. \\
\hline $1-2$ & 0.0192 & 0.0575 & 0.0264 \\
$1-3$ & 0.0452 & 0.1852 & 0.0204 \\
$2-4$ & 0.0570 & 0.1737 & 0.0184 \\
$3-4$ & 0.0132 & 0.0379 & 0.0042 \\
$2-5$ & 0.0472 & 0.1983 & 0.0209 \\
$2-6$ & 0.0581 & 0.1763 & 0.0187 \\
$4-6$ & 0.0119 & 0.0414 & 0.0045 \\
$5-7$ & 0.0460 & 0.1160 & 0.0102 \\
$6-7$ & 0.0267 & 0.0820 & 0.0085 \\
$6-8$ & 0.0120 & 0.0420 & 0.0045 \\
$6-9$ & 0 & 0.2080 & 0 \\
$6-10$ & 0 & 0.5560 & 0 \\
$9-11$ & 0 & 0.2080 & 0 \\
$9-10$ & 0 & 0.1100 & 0 \\
$4-12$ & 0 & 0.2560 & 0 \\
$12-13$ & 0 & 0.1400 & 0 \\
$12-14$ & 0.1231 & 0.2559 & 0 \\
$12-15$ & 0.0662 & 0.1304 & 0 \\
$12-16$ & 0.0945 & 0.1987 & 0 \\
$14-15$ & 0.2210 & 0.1997 & 0 \\
$16-17$ & 0.0824 & 0.1923 & 0 \\
$15-18$ & 0.1070 & 0.2185 & 0 \\
$18-19$ & 0.0639 & 0.1292 & 0 \\
$19-20$ & 0.0340 & 0.0680 & 0 \\
$10-20$ & 0.0936 & 0.2090 & 0 \\
$10-17$ & 0.0324 & 0.0845 & 0 \\
$10-21$ & 0.0348 & 0.0749 & 0 \\
$10-22$ & 0.0727 & 0.1499 & 0 \\
$21-22$ & 0.0116 & 0.0236 & 0 \\
$15-23$ & 0.1000 & 0.2020 & 0 \\
$22-24$ & 0.1320 & 0.2700 & 0 \\
$23-24$ & 0.1320 & 0.2700 & 0 \\
$24-25$ & 0.1885 & 0.3292 & 0 \\
$25-26$ & 0.2544 & 0.3800 & 0 \\
$25-27$ & 0.1093 & 0.2087 & 0 \\
$27-28$ & 0.0000 & 0.3960 & 0 \\
$27-29$ & 0.2198 & 0.4153 & 0 \\
$27-30$ & 0.3202 & 0.6027 & 0 \\
$29-30$ & 0.2399 & 0.4533 & 0 \\
$8-28$ & 0.0636 & 0.2000 & 0.0214 \\
$6-28$ & 0.0169 & 0.0599 & 0.0065 \\
\hline & & &
\end{tabular}


Operation conditions (power in p.u. on 100,000 kVA base) are shown in the following table

\begin{tabular}{|c|c|c|c|c|}
\hline $\begin{array}{l}\text { Bus } \\
\text { number }\end{array}$ & $\begin{array}{l}\text { Voltage } \\
\text { p.u. }\end{array}$ & $\begin{array}{l}\text { Phase angle } \\
\text { p.u. }\end{array}$ & $\begin{array}{l}\text { Real power } \\
\text { p.u. }\end{array}$ & $\begin{array}{l}\text { Imaginary } \\
\text { power p.u }\end{array}$ \\
\hline $1^{\mathrm{a}}$ & 1.06 & 0 & 0 & 0 \\
\hline 2 & 1.045 & 0 & 0.183 & -0.127 \\
\hline 3 & 1.0 & 0 & -0.024 & -0.012 \\
\hline 4 & 1.0 & 0 & -0.076 & -0.016 \\
\hline 5 & 1.01 & 0 & -0.942 & -0.19 \\
\hline 6 & 1.0 & 0 & 0 & 0 \\
\hline 7 & 1.0 & 0 & -0.228 & -0.109 \\
\hline 8 & 1.01 & 0 & -0.300 & -0.300 \\
\hline 9 & 1.0 & 0 & 0 & 0 \\
\hline 10 & 1.0 & 0 & -0.058 & -0.020 \\
\hline 11 & 1.082 & 0 & 0 & 0 \\
\hline 12 & 1.0 & 0 & -0.112 & -0.075 \\
\hline 13 & 1.071 & 0 & 0 & 0 \\
\hline 14 & 1.0 & 0 & -0.062 & -0.016 \\
\hline 15 & 1.0 & 0 & -0.082 & -0.025 \\
\hline 16 & 1.0 & 0 & -0.035 & -0.018 \\
\hline 17 & 1.0 & 0 & -0.090 & -0.058 \\
\hline 18 & 1.0 & 0 & -0.032 & -0.009 \\
\hline 19 & 1.0 & 0 & -0.095 & -0.034 \\
\hline 20 & 1.0 & 0 & -0.022 & -0.007 \\
\hline 21 & 1.0 & 0 & -0.175 & -0.112 \\
\hline 22 & 1.0 & 0 & 0 & 0 \\
\hline 23 & 1.0 & 0 & -0.032 & -0.016 \\
\hline 24 & 1.0 & 0 & -0.087 & -0.067 \\
\hline 25 & 1.0 & 0 & 0 & 0 \\
\hline 26 & 1.0 & 0 & -0.035 & -0.023 \\
\hline 27 & 1.0 & 0 & 0 & 0 \\
\hline 28 & 1.0 & 0 & 0 & 0 \\
\hline 29 & 1.0 & 0 & -0.024 & -0.009 \\
\hline 30 & 1.0 & 0 & -0.106 & -0.019 \\
\hline
\end{tabular}

${ }^{\mathrm{a}}$ Swing bus.
The following table shows transformer data:

\begin{tabular}{rl}
\hline Transformer designation & Tap setting $^{\mathrm{a}}$ \\
\hline $4-12$ & 0.932 \\
$6-9$ & 0.978 \\
$6-10$ & 0.969 \\
$28-27$ & 0.968 \\
\hline
\end{tabular}

${ }^{\text {a }}$ Off-normal turn ratio, as determined by the actual transformer-tap positions and the voltage bases. In the case, the normal turn ratio equals 1.

Static capacitor data (suceptance in p.u. on 100,000 kVA base) are as follows:

\begin{tabular}{ll}
\hline Bus number & Susceptance p.u. \\
\hline 10 & 0.19 \\
24 & 0.043
\end{tabular}

\section{References}

[1] Available transfer capability definition and determination. NERC, June 1996

[2] MacArthur CA. Transmission limitations computed by superposition. AIEE Trans. 1961;December:827-31.

[3] Ilic MD, Yoon YT, Zobian A. Available transmission capacity (ATC) and its value under open access. IEEE Trans. on Power Systems 1997;12(2):636-45.

[4] Wu FF, Kumagai S. Steady state security regions of power systems. IEEE Trans. on CAS 1982;29(11):703-11.

[5] Marceau RJ, Malihot R, Galiana FD. A generalized shell for dynamic security analysis in operations planning. IEEE Trans. on Power Systems 1993;8(3):1099-106.

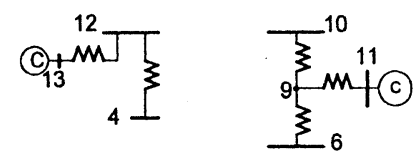

C) synchronous compensator

(G) generators

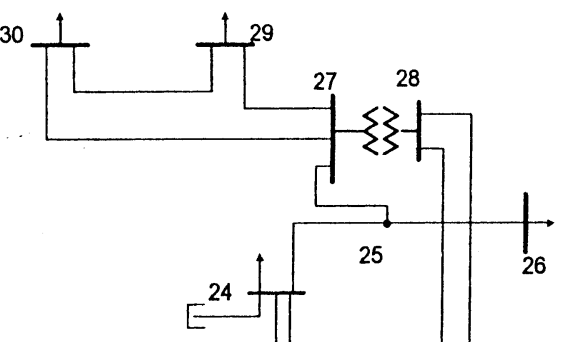

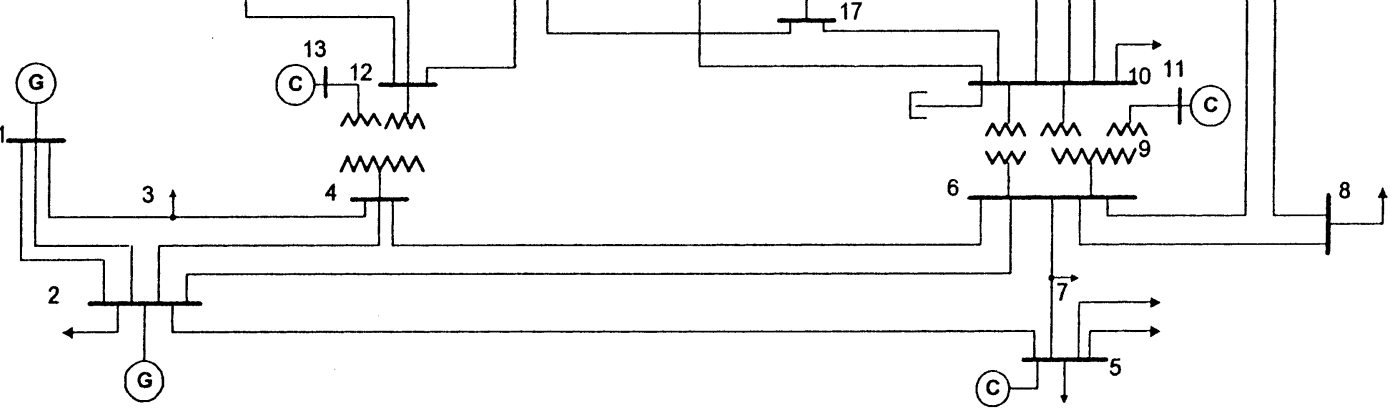


[6] Demaree K, Athay T, Cheung K, Mansour Y, Vaahedi E, Chang AY, Corns BR, Garrett BW. An on-line dynamic security analysis system implementation. IEEE Trans. on Power Systems 1994;9(4): 1716-22.

[7] Mansour Y, Vaahedi E, Chang AY, Corns BR, Garrett BW, Demaree K, Athay T, Cheung K. Hydro's on line transient stability assessment (TSA) model development, analysis and post-processing. IEEE Trans. on Power Systems 1995;10(1):241-53.

[8] Shirmohammadi D, Gribik PR, Law ETK, Malinowski JH, O’Donnell RE. Evaluation of transmission network capacity use for wheeling transaction. IEEE Trans. on Power Systems 1989;4(4):1405-13.

[9] Galiana F, Banakar M. Security corridors in power systems. IEEE Trans. on PAS 1981;100(3):1128-37.

[10] Wunderlich S, Wu T, Fischl R, O'Connell R. An inter-area transmission and voltage limitation (TVLIM) program. IEEE Trans. on Power Systems 1995;10(3):1257-63.

[11] Peschon J, Piercy DS, Tinney WF, Tveit OJ. Sensitivity in power systems. IEEE Trans. on PAS 1968;87(8):1687-96.

[12] Gomez A, Martinez JL, Ruiz JL, Cuellar Y. Sensitivity based reactive power control for voltage profile improvement. IEEE Trans. on Power Systems 1993;8(3):937-45.

[13] Gibescu M, Christie RD. Quadratic sensitivities for power system steady state control. IEE Proc. Gener. Transm. Distrib. 1997;144(3):317-22.

[14] Desoer CA, Kuh ES. Basic Circuit Theory, McGraw-Hill, 1969. p. 385.

[15] Hasler M, Wang C, Ilic M, Zobian A. Computation of static stability margins in power systems using monotonicity. IEEE ISCAS Proc. 1993:2196-9.

[16] Ilic M. Network theoretic condictions for existence and uniqueness of steady state solutions to electric power circuits. IEEE ISCAS Proc. 1992:2821-8.

[17] Chauffoureaux P, Hasler M. Monotonicity in nonlinear resistive circuits IEEE ISCAS Proc. 1990:395-8.

[18] Freris LL, Sasson AM. Investigation of the load flow problem. IEE Proc. 1968;115(10):1459-70.

[19] Ilic M, Galiana F, Fink L, Bose A, Mallet P, Othman H. Transmission capacity in power networks. Electrical Power \& Energy Systems 1998;20(2):99-110. 